\title{
Nicorandil effects on platelet function, Hs-CRP, MMP-9 and myocardial antioxidation in patients with unstable angina
}

\author{
RUIJUN PENG, XILI YANG and XI LIANG \\ Department of Cardiovascular Medicine, The First People's Hospital of FoShan \\ (Affiliated FoShan Hospital of Sun Yat-sen University), Foshan, Guangdong 528000, P.R. China
}

Received June 26, 2018; Accepted May 10, 2019

DOI: $10.3892 /$ etm.2019.7918

\begin{abstract}
Nicorandil effects on platelet activation and myocardial antioxidant function in patients with unstable angina were studied. A total of 157 patients with unstable angina in the First People's Hospital of Foshan were selected and divided into experimental and control group. Patients in experimental group were treated with conventional drugs and nicorandil (15 min/day), t.i.d., for 21 days as one course of treatment, while those in control group were treated with conventional drugs. After treatment, serum indexes were detected and compared between the two groups of patients. Compared with that in control group, the platelet function of patients in the experimental group was significantly improved, and there was a statistically significant difference $(\mathrm{P}<0.05)$. Serum antioxidation factors in both groups were increased after treatment, and they were increased more significantly in experimental group $(\mathrm{P}<0.05)$. Serum inflammatory factors, high-sensitivity C-reactive protein and matrix metalloproteinase-9, also declined significantly in the experimental group. Nicorandil reduces inflammatory response and promotes stability of myocardial function in the treatment of unstable angina.
\end{abstract}

\section{Introduction}

Unstable angina is a common disease in clinic, which frequently occurs in the middle-aged and elderly people, and will easily develop into myocardial infarction if not treated in time. In recent years, with the increase of social pressure on middle-aged people and the increase of aging population, the incidence rate of unstable angina has also shown an increasing trend year by year. Studies have also revealed that angina is possibly related to obesity and familial inheritance $(1,2)$.

Correspondence to: Dr Xili Yang, Department of Cardiovascular Medicine, The First People's Hospital of FoShan (Affiliated FoShan Hospital of Sun Yat-sen University), 81 North Lingnan Avenue, Foshan, Guangdong 528000, P.R. China

E-mail:wrly479@163.com

Key words: unstable angina, platelet activation, myocardial antioxidation, nicorandil, C-reactive protein
Coronary atherosclerosis can lead to stenosis of vascular lumen, and ischemic necrosis occurs easily in the myocardium with underdeveloped collateral circulation, thus resulting in myocardial infarction. Atheromatous plaques are mainly composed of cholesterol and cholesteryl ester characterized by high brittleness, which, under the influence of heart rate, blood pressure and myocardial contractility, will rupture or fall off easily and flow with the blood, blocking small blood vessels or capillaries, forming thrombus and aggravating myocardial infarction (3-5). Currently, conservative medical therapy is dominated in the treatment of unstable angina, such as anti-platelet aggregation, dilatation of coronary artery and lipid lowering. According to the research on pathogenesis of unstable angina, the inflammatory response-induced decline in the stability of atherosclerotic plaque, platelet activation and aggregation are important conditions causing the disease, so alleviating inflammatory response, improving plaque stability and inhibiting platelet activation are important measures to prevent the progression of unstable angina and reduce the risk of myocardial infarction $(6,7)$.

Nicorandil is a nitrate drug with an effect of opening potassium ion channel possessing anti-inflammatory, antioxidation and endothelium protective functions (8). Therefore, it has been widely applied in the treatment after interventional operation of coronary heart disease, achieving positive curative effects (9). However, the value of nicorandil in the treatment of unstable angina remains unclear. Thus, the effects of nicorandil on inflammatory factors, platelet and myocardial antioxidant function in patients with unstable angina were studied.

\section{Patients and methods}

Basic data. A total of 157 patients diagnosed with unstable angina in the First People's Hospital of FoShan (Affiliated FoShan Hospital of Sun Yat-sen University; Foshan, China) from 2012 to 2017 were selected (10). The patients were numbered as 1, 2, 3...157 and then divided into even and odd number group, then further defined as experimental and control group, respectively. Considering that several patients in the control group were unstable angina pectoris accompanied with myocardial infarction, these patients were included in the experimental group. Finally, 73 patients in the control group and 84 patients in the experimental group were included. Basic 
Table I. Comparison of basic data between the two groups of patients.

\begin{tabular}{lcccc}
\hline Item & $\begin{array}{c}\text { Control } \\
\text { group }(\mathrm{n}=73)\end{array}$ & $\begin{array}{c}\text { Experimental } \\
\text { group }(\mathrm{n}=84)\end{array}$ & $\mathrm{t} / \mathrm{F} / \chi^{2}$-value & P-value \\
\hline Sex (male/female) & $34 / 39$ & $34 / 50$ & 0.55 & 0.36 \\
Age (years) & $56.7 \pm 11.5$ & $58.6 \pm 10.8$ & 2.19 & 0.76 \\
Body mass index $\geq 25 \mathrm{~kg} / \mathrm{m}^{2}$ & 27 & 33 & 0.02 & 0.76 \\
Familial genetic history & 15 & 23 & 0.65 & 0.31 \\
Basic disease & & & & 0.31 \\
Myocardial infarction & 34 & 45 & 2.26 & 0.09 \\
Coronary heart disease & 32 & 48 & 1.12 & 0.86 \\
Hypertension & 26 & 31 & 0.43 & 0.36 \\
Liver disease & 7 & 6 & 0.009 & 0.67 \\
Diabetes & 4 & & & \\
\hline
\end{tabular}

data, such as age, sex, familial genetic history, obesity index and underlying complications, including diseases associated with unstable angina, including myocardial infarction, coronary heart disease, hypertension, liver diseases (fatty liver, high cholesterol and gallstone) and diabetes, were comparable between the two groups of patients $(\mathrm{P}>0.05)$.

The study was approved by the Ethics Committee of the First People's Hospital of FoShan (Affiliated FoShan Hospital of Sun Yat-sen University). Patients who participated in this research had complete clinical data. The signed informed consents were obtained from the patients or the guardians.

Treatment methods. At the beginning of treatment, patients in both groups were orally administered conventional drugs for angina, including aspirin tablets + metoprolol + atorvastatin + isosorbide dinitrate. Patients were asked to take nitroglycerin under tongue at the onset of angina. Hypertension patients were given antihypertensive drugs, and hyperlipidemia patients took statins for lipid-lowering treatment. Patients in the experimental group, based on conventional drug treatment, were orally administered nicorandil tablets (15 mg/day, 5 mg/tablet, H20080418, Asahi Kasei Corporation), t.i.d., for 21 days as one course of treatment.

Observation indexes. Before and after treatment, venous blood was collected and EDTA was added for anticoagulation. Then the blood was centrifuged at $2,000 \times \mathrm{g}$ for $10 \mathrm{~min}$ at $4^{\circ} \mathrm{C}$. Changes in levels of matrix metalloproteinase-9 (MMP-9; ELISA kit), high-sensitivity C-reactive protein (hs-CRP; C-SPOT kit), superoxide dismutase (SOD; ELISA kit) and malondialdehyde (MDA; C-SPOT kit) in the supernatant were detected via enzyme-linked immunosorbent assay (ELISA). The platelet function was detected through labeling and screening platelet fluorescence antibodies, such as CD63, CD42b and GP-VI. Moreover, parameters in thromboelastogram, such as coagulation response time (R), formation time of coagulation block $(\mathrm{K})$, thrombosis rate (Angle), comprehensive coagulation index (CI) and maximum amplitude (MA), were measured using a coagulation analyzer (TEG-5000, Haemonetics). Angle represents the rate angle of hemocyte agglutination, which indicates the angle between tangent line and horizontal line from the forming point to the maximum curve Radian of the marker map. MA represents the maximum strength or hardness of the clot and the stable form of the clot. All experimental operations were carried out by specialized technicians in strict accordance with the steps of the kit, and the experimental data were repeated three times to take the average value.

MMP-9 and hs-CRP as anti-inflammatory factor in the blood can reflect the occurrence and level of inflammation in the blood. SOD and MDA are reductive substances in cells representing the strength of antioxidant capacity of cardiomyocytes. Coagulation reaction time, clotting time, thrombus formation rate, synthetic coagulation index and maximum amplitude were the parameters of platelet coagulation function. These parameters were selected to represent the levels of inflammation, myocardial antioxidant capacity and platelet function in patients with unstable angina pectoris. These parameters were easy to detect, and had little damage to the patients.

Statistical analysis. SPSS17.0 (SPSS Inc., Chicago, IL, USA) was used for statistical analysis. Chi-square test was used for counting data and paired t-test was used for measuring data. $\mathrm{P}<0.05$ was considered as the difference with statistical significance.

\section{Results}

Comparison of basic data between the two groups of patients. Basic data, including sex, age, body mass index, familial genetic history, basic disease had no statistically significant differences between the two groups of patients, and they were comparable $(\mathrm{P}>0.05)$ (Table I).

Comparison of platelet function between the two groups of patients. After treatment, the platelet function was compared between the two groups of patients. Results revealed that expression of platelet membrane glycoproteins GP-VI, CD42b, PAC-1 and CD63 in the experimental group was $31.7 \pm 6.72,27.8 \pm 5.33$, $21.54 \pm 3.28$ and $36.7 \pm 8.25$, respectively, which is lower than those in control group $(\mathrm{P}<0.05)$, while $\mathrm{R}$ and $\mathrm{K}$ in the experimental 
Table II. Comparison of platelet parameters between the two groups of patients.

\begin{tabular}{lllll}
\hline Groups & GP-VI & CD42b & PAC-1 & CD63 \\
\hline Control (n=73) & $77.5 \pm 13.64$ & $49.6 \pm 7.29$ & $41.7 \pm 8.59$ & $63.16 \pm 9.27$ \\
Experimental $(\mathrm{n}=84)$ & $31.7 \pm 6.72^{\mathrm{a}}$ & $27.8 \pm 5.33^{\mathrm{a}}$ & $21.54 \pm 3.28^{\mathrm{a}}$ & $36.7 \pm 8.25^{\mathrm{a}}$ \\
\hline
\end{tabular}

GP-VI, glycoprotein VI. PAC, platelet-associated complement. ${ }^{\mathrm{a}} \mathrm{P}<0.05$, compared with control group.

Table III. Comparison of serum hs-CRP and MMP-9 levels between the two groups of patients.

\begin{tabular}{lccccc}
\hline & & hs-CRP $(\mathrm{ng} / \mathrm{ml})$ & & \multicolumn{1}{c}{$\mathrm{MMP}-9(\mathrm{pg} / \mathrm{ml})$} \\
Groups & Before treatment & After treatment & & Before treatment & After treatment \\
\hline Control $(\mathrm{n}=73)$ & $16.36 \pm 1.33$ & $9.14 \pm 1.23$ & & $227.63 \pm 36.81$ & $82.9 \pm 9.48$ \\
Experimental $(\mathrm{n}=84)$ & $16.87 \pm 1.54$ & $6.07 \pm 0.94^{\mathrm{a}}$ & & $233.73 \pm 32.55$ & $49.2 \pm 6.22^{\mathrm{a}}$ \\
\hline
\end{tabular}

${ }^{a} \mathrm{P}<0.05$, compared to control group; MMP-9, matrix metalloproteinase-9; hs-CRP, high-sensitivity C-reactive protein.
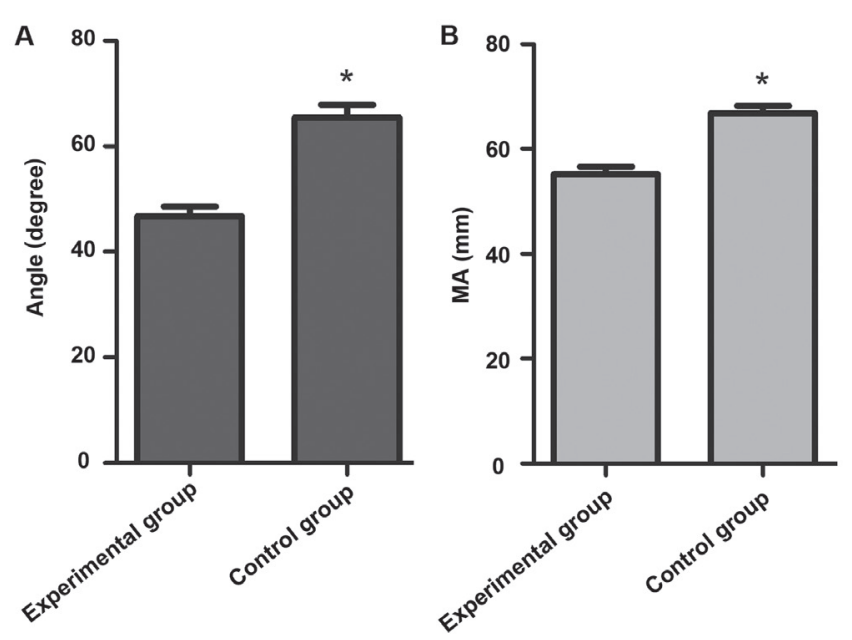

Figure 1. Comparison of Angle and MA in thromboelastogram between the two groups of patients. Angle (A) and MA (B) in experimental group were lower than those in control group $\left({ }^{*} \mathrm{P}<0.05\right)$. Angle, thrombosis rate; MA, maximum amplitude.

group were longer than those in control group, showing statistically significant differences $(\mathrm{P}<0.05)$. The above indicates that the experimental group can inhibit better the activation and the platelet function (Table II and Figs. 1 and 2).

Comparison of serum hs-CRP and MMP-9 levels between the two groups of patients. After treatment for 21 days, levels of serum hs-CRP and MMP-9 in both groups of patients declined compared with those before treatment. Levels of $(6.07 \pm 0.94)$ and MMP-9 (49.2 \pm 6.22$)$ in the experimental group declined more significantly than those in control group $(\mathrm{t}=12.93$ and $8.95, \mathrm{P}<0.05)$, and differences were statistically significant, indicating that nicorandil can significantly reduce levels of serum hs-CRP and MMP-9 in patients with unstable angina and inhibited the activation of platelet by serum inflammatory factors (Table III).

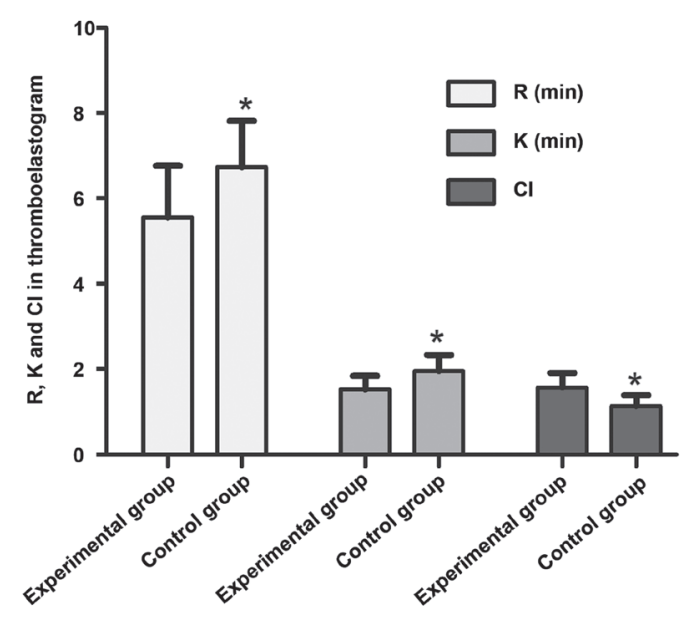

Figure 2. Comparison of R, K and CI in thromboelastogram between the two groups of patients. $\mathrm{R}$ and $\mathrm{K}$ in experimental group were lower than those in control group and CI in the experimental group was higher than that in control group $\left({ }^{*} \mathrm{P}<0.05\right)$. $\mathrm{R}$, coagulation response time; $\mathrm{K}$, formation time of coagulation block; $\mathrm{CI}$, comprehensive coagulation index.

Comparison of myocardial antioxidant function between the two groups of patients. Levels of serum anti-oxidation molecules MDA and SOD were compared between the two groups of patients after treatment. Results manifested that the serum SOD level was increased, while the MDA level declined (results are not shown) in both groups compared with those before treatment. Changes in levels of anti-oxidation molecules were more significant in the experimental group compared with those in control group $(\mathrm{P}<0.05)$ (Fig. 3).

\section{Discussion}

Stable angina will develop into unstable angina with the increased frequency of pain, increase of precipitating factors and progressive aggravation due to a variety of factors $(11,12)$. Due to the instability of local coronary artery lesions in patients 
A

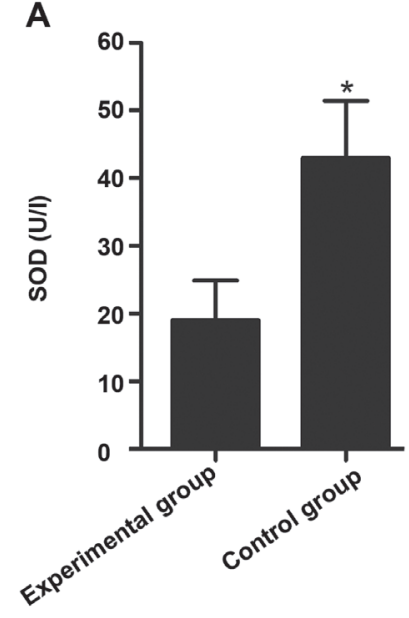

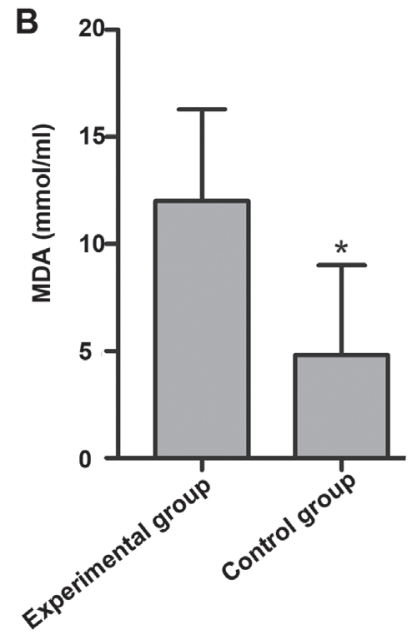

Figure 3. Comparison of myocardial antioxidant function between the two groups of patients. (A) Serum SOD level was increased in both groups compared with those before treatment. (B) MDA level declined (results are not shown) in both groups compared with those before treatment. SOD, superoxide dismutase; MDA, malondialdehyde. ${ }^{*} \mathrm{P}<0.05$.

and other factors, the frequency, degree and precipitating factors of angina are often changed, the pain will aggravate or return to stability, and unstable angina may develop into myocardial infarction in late stage (13), affecting the normal life and work of patients. Currently, conservative medical therapy dominates the treatment of unstable angina, including anticoagulation therapy, inhibition of platelet aggregation and activation, lipid-regulating drugs, blood pressure lowering, thrombolytic therapy and dilation of blood vessels. High-risk patients may also be treated with emergency interventional therapy or emergency coronary artery bypass grafting (14-16). In conservative medical therapy, nicorandil is often applied as an adjuvant drug for unstable angina, which, as a nitrate drug with an effect of opening potassium ion channel, it can reduce the level of calcium ion through activating guanylate-activating enzymein cells, thereby relaxing the vascular smooth muscle and dilating the coronary artery $(17,18)$. In addition, nicorandil also possesses anti-inflammatory, anti-oxidation and endothelium protective functions. However, nicorandil has adverse reactions such as inducing mucosal ulcer, and it easily leads to hemorrhage and infection in patients $(19,20)$, so there is still a certain risk in the clinical application of nicorandil in the treatment of unstable angina, and further in-depth research is needed.

The occurrence and development of unstable angina is a complex process involving a variety of predisposing factors. Atherosclerotic plaque, myocardial ischemia and viscous blood lipid can induce unstable angina, in which platelet activation and aggregation play key roles. After the rupture of atherosclerotic plaque, the collagen under the vascular endothelium is exposed, activating and aggregating platelets. Platelets aggregate around the plaque and release a large number of inflammatory factors, accelerating the formation of thrombus, leading to stenosis of coronary arterial lumen and reducing the blood flow, thereby resulting in insufficient myocardial blood supply. At the same time, platelets will also release inflammatory factors into the blood after activation, and then these inflammatory factors can change the texture and stability of atherosclerotic plaque, induce the rupture of atherosclerotic plaque, and promote

the formation of thrombus. In addition, oxygen free radicals can also induce the expression of inflammatory cytokines in endothelial cells, increasing the incidence of cardiovascular and cerebrovascular diseases. SOD can scavenge the superoxide ion by disproportionation and reduce the damage of the endothelial cells caused by oxygen free radicals. Therefore, anti-inflammation, and inhibition of platelet activation and reducing oxygen free radicals are the most important links in the treatment process of patients with unstable angina. This study investigated the effects of nicorandil on platelet activation, inflammatory factors and myocardial antioxidant function in patients with unstable angina, so as to provide clinical data support for the treatment of patients with unstable angina.

Collectively, it was found that expression of platelet membrane glycoproteins CD63, PAC-1, CD42b and GP-VI obviously declined in patients taking nicorandil, and $\mathrm{R}$ and $\mathrm{K}$ in peripheral blood were longer than those in patients who did not take nicorandil, while Angle, MA, and CI were lower than those in patients who did not take nicorandil, indicating that nicorandil can inhibit platelet activation and reduce thrombosis rate and capacity in patients with unstable angina. Moreover, results of blood biochemical study revealed that the decline in the content of serum CRP and MMP-9 in experimental group was more significant than that in control group, suggesting that nicorandil has a certain effect of reducing the inflammatory response in patients with unstable angina. In the analysis of myocardial antioxidant function of patients, changes in levels of anti-oxidation factors MDA and SOD were also compared between experimental group and control group. Results manifested that after treatment, the level of SOD in the experimental group was higher than that in the control group, and the decreasing level of MDA was also higher than that in the control group $(\mathrm{P}<0.05)$, indicating that nicorandil has a certain effect of increasing myocardial anti-oxidation factors, improving myocardial anti-oxidation level and protecting myocardium. At present, nicorandil can be used not only in patients with hypertension, but also in patients with angina pectoris, but the therapeutic effect of nicorandil in patients with unstable colic needs to be further studied. The side effect of nicorandil is mainly headache, dizziness and other adverse reactions. The comprehensive drug effect and metabolism in patients with unstable angina pectoris are worthy of further study.

In conclusion, nicorandil can help reduce inflammatory response, inhibit platelet function, increase myocardial antioxidation level and improve therapeutic efficacy of patients with unstable angina, which is worthy of further promotion and application in clinic.

\section{Acknowledgements}

Not applicable.

\section{Funding}

No funding was received.

\section{Availability of data and materials}

The datasets used and/or analyzed during the current study are available from the corresponding author on reasonable request. 


\section{Authors' contributions}

RP wrote the manuscript and collected the basic information of patients. XY and XL recorded and analyzed observation indexes. All the authors read and approved the final manuscript.

\section{Ethics approval and consent to participate}

The study was approved by the Ethics Committee of the First People's Hospital of FoShan (Affiliated FoShan Hospital of Sun Yat-sen University; Foshan, China). Patients who participated in this research had complete clinical data. The signed informed consents were obtained from the patients or the guardians.

\section{Patient consent for publication}

Not applicable.

\section{Competing interests}

The authors declare that they have no competing interests.

\section{References}

1. MacMahon S, Sharpe N, Gamble G, Hart H, Scott J, Simes J and White H; LIPID Trial Research Group: Effects of lowering average of below-average cholesterol levels on the progression of carotid atherosclerosis: Results of the LIPID Atherosclerosis Substudy. Circulation 97: 1784-1790, 1998.

2. Hauser ER, Mooser V, Crossman DC, Haines JL, Jones CH, Winkelmann BR, Schmidt S, Scott WK, Roses AD, Pericak-Vance MA, et al; Design of the Genetics of Early Onset Cardiovascular Disease study: Design of the Genetics of Early Onset Cardiovascular Disease (GENECARD) study. Am Heart J 145: 602-613, 2003.

3. Vasilieva EJ, Shpector AV, Raskuragev AB, Lekochmacher SS and Bespalko IA: Platelet function and plasma lipid levels in patients with stable and unstable angina pectoris. Am J Cardiol 68: 959-961, 1991.

4. Ogawa H, Yasue H, Misumi I, Masuda T, Okumura K, Bannai S, Takanashi $\mathrm{N}$ and Tsukada Y: Plasma platelet-derived growth factor levels in coronary circulation in unstable angina pectoris. Am J Cardiol 69: 453-456, 1992.

5. Chakhtoura EY, Shamoon FE, Haft JI, Obiedzinski GR, Cohen AJ and Watson RM: Comparison of platelet activation in unstable and stable angina pectoris and correlation with coronary angiographic findings. Am J Cardiol 86: 835-839, 2000.
6. Fitzgerald DJ: Platelet activation in the pathogenesis of unstable angina: Importance in determining the response to plasminogen activators. Am J Cardiol 68: 51B-57B, 1991.

7. Fitzgerald DJ, Roy L, Catella F and FitzGerald GA: Platelet activation in unstable coronary disease. N Engl J Med 315: 983-989, 1986.

8. Frampton J, Buckley MM and Fitton A: Nicorandil. A review of its pharmacology and therapeutic efficacy in angina pectoris. Drugs 44: 625-655, 1992.

9. Kim JH, Jeong MH, Yun KH, Kim KH, Kang DK, Hong SN, Lim SY, Lee SH, Lee YS, Hong YJ, et al: Myocardial protective effects of nicorandil during percutaneous coronary intervention in patients with unstable angina. Circ J 69: 306-310, 2005.

10. Natarajan M: Unstable angina. Clin Evid 8: 225-235, 2002.

11. Xu FH and Wang JM: Clinical observation on acupuncture combined with medication for intractable angina pectoris Zhongguo Zhenjiu 25: 89-91, 2005 (In Chinese).

12. Tada M: Unstable angina. Nippon Naika Gakkai Zasshi 72: 721-726, 1983 (In Japanese).

13. Ibuki C: Unstable angina. Nihon Rinsho 2 (Suppl 5): 234-239, 2007 (In Japanese).

14. Waters DD, Walling A, Roy D and Théroux P: Previous coronary artery bypass grafting as an adverse prognostic factor in unstable angina pectoris. Am J Cardiol 58: 465-469, 1986.

15. Faxon DP, Detre KM, McCabe CH, Fisher L, Holmes DR, Cowley MJ, Bourassa MG, Van Raden M and Ryan TJ: Role of percutaneous transluminal coronary angioplasty in the treatment of unstable angina. Report from the National Heart, Lung, and Blood Institute Percutaneous Transluminal Coronary Angioplasty and Coronary Artery Surgery Study Registries. Am J Cardiol 53: 131C-135C, 1984.

16. Lubsen J: Medical management of unstable angina. What have we learned from the randomized trials? Circulation 82 (Suppl): II82-II87, 1990.

17. Walker A, McMurray J, Stewart S, Berger W, McMahon AD, Dargie H, Fox K, Hillis S, Henderson NJ and Ford I: Economic evaluation of the impact of nicorandil in angina (IONA) trial. Heart 92: 619-624, 2006.

18. Kim SJ, Kim W, Woo JS, Ha SJ, Kang WY, Hwang SH, Kang DG, Lee SU, Cho SK, Im JS, et al: Effect of myocardial protection of intracoronary adenosine and nicorandil injection in patients undergoing non-urgent percutaneous coronary intervention: A randomized controlled trial. Int J Cardiol 158: 88-92, 2012.

19. Egred M: Nicorandil-associated ulcerations. Eur J Gastroenterol Hepatol 19: 395-398, 2007.

20. Gupta A and Morris G: Major aphthous ulcers induced by nicorandil. Age Ageing 29: 372-373, 2000.

(i) $\ominus$ This work is licensed under a Creative Commons

EY NO ND Attribution-NonCommercial-NoDerivatives 4.0 International (CC BY-NC-ND 4.0) License. 